\title{
Disease specific characteristics of fetal epigenetic markers for non-invasive prenatal testing of trisomy 21
}

\author{
Ji Hyae Lim ${ }^{1 \dagger}$, Da Eun Lee ${ }^{1 \dagger}$, So Yeon Park', Do Jin Kim ${ }^{1}$, Hyun Kyong Ahn², You Jung Han², Moon Young Kim² \\ and Hyun Mee Ryu ${ }^{1,2^{*}}$
}

\begin{abstract}
Background: Non-invasive prenatal testing of trisomy 21 (T21) is being actively investigated using fetal-specific epigenetic markers (EPs) that are present in maternal plasma. Recently, 12 EPs on chromosome 21 were identified based on tissue-specific epigenetic characteristics between placenta and blood, and demonstrated excellent clinical performance in the non-invasive detection of fetal T21. However, the disease-specific epigenetic characteristics of the EPs have not been established. Therefore, we validated the disease-specific epigenetic characteristics of these EPs for use in non-invasive detection of fetal T21.

Methods: We performed a high-resolution tiling array analysis of human chromosome 21 using a methyl-CpG binding domain-based protein (MBD) method with whole blood samples from non-pregnant normal women, whole blood samples from pregnant normal women, placenta samples of normal fetuses, and placenta samples of T21 fetuses. Tiling array results were validated by bisulfite direct sequencing and qPCR.

Results: Among 12 EPS, only four EPs were confirmed to be hypermethylated in normal placenta and hypomethylated in blood. One of these four showed a severe discrepancy in the methylation patterns of T21 placenta samples, and another was located within a region of copy number variations. Thus, two EPs were confirmed to be potential fetal-specific markers based on their disease-specific epigenetic characteristics. The array results of these EPs were consisted with the results obtained by bisulfite direct sequencing and qPCR. Moreover, the two EPs were detected in maternal plasma.

Conclusions: We validated that two EPs have the potential to be fetal-specific EPs which is consistent with their disease-specific epigenetic characteristics. The findings of this study suggest that disease-specific epigenetic characteristics should be considered in the development of fetal-specific EPs for non-invasive prenatal testing of T21.
\end{abstract}

Keywords: Trisomy 21, Non-invasive prenatal testing, Epigenetic markers

\section{Background}

Prenatal testing is an integral component of modern obstetric practice and is commonly performed in professional medical organizations worldwide. The primary aim of prenatal testing is the diagnosis of fetal aneuploidies, such as trisomy 21 (T21, Down syndrome), trisomy 18

\footnotetext{
* Correspondence: hmryu@yahoo.com

${ }^{\dagger}$ Equal contributors

'Laboratory of Medical Genetics, Medical Research Institute, Cheil General

Hospital and Women's Healthcare Center, Seoul, Korea

${ }^{2}$ Department of Obstetrics and Gynecology, Cheil General Hospital and

Women's Healthcare Center, Kwandong University College of Medicine, Seoul, Korea
}

(Edwards syndrome) and trisomy 13 (Patau syndrome) [1]. Although the majority of fetuses with aneuploidy result in spontaneous termination during fetal development, T21 has the highest survival rate, affecting 1 in 800 births [2]. Therefore, prenatal detection of T21 is considered the most common and important aspect of prenatal genetic testing. Current prenatal screening tests of T21 have greatly improved by using a combination of maternal serum markers and fetal sonographic markers such as nuchal translucency [3-6]. However, positive screening results require confirmation with diagnostic testing, such as amniocentesis or chorionic villus sampling (CVS) [7]. 
However, both sampling procedures are invasive, and are associated with significant risks to the fetus and mother, including the potential loss of a healthy fetus $[7,8]$. For this reason, invasive prenatal diagnostic tests are currently preformed only in high-risk pregnancies or in pregnancies with increased maternal age and/or family history of having a child with an inherited disease. Therefore, developing a reliable method for non-invasive prenatal testing (NIPT) for fetal T21 is of critical importance in prenatal care.

To perform NIPT, a source of fetal genetic material that could be sampled without harm to the fetus is needed. In 1997, Lo et al. discovered the existence of cell-free fetal DNA (cff-DNA) in maternal circulation [9]. It is rapidly cleared from maternal blood within two hours of delivery and constitutes approximately $10 \%$ of the total DNA in maternal plasma [10,11]. Moreover, it has recently been found that the entire fetal genome, in the form of cff-DNA, is present in maternal blood [12]. Therefore, cff-DNA is regarded as a promising new material for the development of reliable NIPT of fetal T21.

Various methods have been applied for the NIPT of fetal T21 using cff-DNA. Recently, the NIPT for T21 based on next-generation sequencing reach sensitivities and specificities of over $99 \%$ and have already been applied in the clinical setting [13-15]. However, nextgeneration sequencing technologies are of high cost and not easily accessible to diagnostic laboratories, because requires major and expensive infrastructure. To overcome these issues, epigenetic modifications as fetal-specific signatures to detect cff-DNA from circulating maternal DNA have been investigated. A number of fetal-specific epigenetic markers (EPs) on chromosome 21 that could be used for the NIPT of fetal T21 have been reported. However, the diagnostic accuracy of these fetal-specific EPs for the NIPT of fetal T21 range from 80 to $100 \%$, depending on the markers and methods used [16-18].

In a previous study, Papageorgiou et al. identified 12 tissue-specific differentially methylated regions (DMRs) that are hypermethylated in placental DNA of the euploid fetus and hypomethylated in non-pregnant female normal whole blood DNA using methylated DNA immunoprecipitation and tiling microarray [19]. The DMRs were suggested for use as fetal-specific EPs in the NIPT of fetal T21. They proposed a fetal-specific DNA methylation ratio method using the EPs for fetal T21 detection and reported excellent clinical performance (both the sensitivity and specificity were 100\%) [18]. However, the EPs were selected based on the only tissue-specific epigenetic characteristics between placenta and blood and their diseasespecific epigenetic characteristics have not been validated.

The aim of this study was to validate disease-specific epigenetic characteristics of EPs for the NIPT of fetal T21 in whole blood from non-pregnant euploid women, whole blood from pregnant euploid women, euploid fetal placenta, and T21 fetal placenta using a high-resolution tiling array analysis of human chromosome 21 and to select the effective EPs that have the potential to be used as fetal-specific EPs for the NIPT of fetal T21.

\section{Methods \\ Ethics statement}

This study was conducted according to the principles expressed in the Declaration of Helsinki. Appropriate institutional review board approval for this study was obtained from the Ethics Committee at Cheil General Hospital (\#CGH-IRB-2011-85). All patients provided written informed consent for the collection of samples and subsequent analysis.

\section{Sample processing}

Pregnant women with euploid or T21 fetuses and nonpregnant euploid women who attended the Department of Obstetrics and Gynecology at Cheil General Hospital, Korea were recruited between March and December 2011. Maternal peripheral blood samples were collected into tubes containing EDTA just before obstetric procedures (such as CVS) in the first trimester. All placenta samples were obtained at CVS. Maternal peripheral blood samples were centrifuged at $1,600 \mathrm{~g}$ for $10 \mathrm{~min}$. The peripheral blood cell portion was re-centrifuged at 2,500 g for $10 \mathrm{~min}$, and any residual plasma was removed. DNA was extracted from maternal peripheral blood cells using the QIAamp Blood kit (Qiagen, West Sussex, UK) and from placental tissues using the QIAamp Tissue Kit (Qiagen).

\section{Array hybridizations}

Whole blood samples from non-pregnant euploid women, whole blood samples from pregnant euploid women, euploid fetal placenta samples, and T21 fetal placenta samples from pregnant euploid women were used. Each DNA sample was extracted from placental tissues and maternal blood cells, and then sonicated and subjected to a methyl-CpG binding domain-based protein (MBD) method. The subsequent product was amplified and labeled using a Genomic DNA Enzymatic Labeling Kit (Agilent Technologies, DE, USA) and hybridized to a customized chip specific for chromosome 21 . The array platforms were composed of 0.4 million 50 -mer to 60-mer oligonucleotides covering chromosome 21 at a median probe density of 1 per $60 \mathrm{bp}$. Microarray protocols, including labeling, hybridization and post-hybridization washing procedures, were performed as recommended by the manufacturer and are available at https://www.home.agilent.com. Briefly, the labeled MBD (Cyanine 5-dUTP: Cy5) DNA and reference DNA (Cyanine 3-dUTP: Cy3) were cleaned using Amicon filters (Millipore Corporation, MA, USA). Competitive hybridization onto the microarray was then performed using a 
Methylation Hybridization Kit (Agilent Technologies) in a rotating SureHyb chamber at $67^{\circ} \mathrm{C}$ for $40 \mathrm{~h}$ according to the manufacturer's instructions. Washed slides were scanned using a High-Resolution C Scanner (Agilent Technologies) and fluorescence was evaluated using Feature Extraction software (Agilent Technologies).

\section{Data analysis of the tiling oligonucleotide arrays}

The two signal values were normalized using background subtraction, and signal ratio (MBD/input), signal log ratio $\left[\log _{2}(\mathrm{MBD} /\right.$ input $\left.)\right], \mathrm{P}[\mathrm{X}]$, and $\mathrm{P}$ were obtained using Agilent Genomic Workbench software (Agilent Technologies). The $\log _{2}$ value is the $\mathrm{Cy} 5: \mathrm{Cy} 3$ fluorescence ratio (methylated DNA recovered by MBD capture: total input DNA) for each probe, converted to a $\log _{2}$ scale, and represents a relative measure of the amount of methylated DNA at each locus. We applied median and Lowess normalization to the raw data and filtered outlier probes to remove low-quality data points. The $\mathrm{P}[\mathrm{X}]$ and $\mathrm{P}$ values, which are used in the MBD array analysis to obtain a binding call, were defined as the probability that the $\mathrm{X}$ value deviates from the Gaussian distribution of $\mathrm{X}$ values of the entire genome of a sample. Here, the $\mathrm{X}$ value for a probe was the difference between the MBD and the input signals after adjusting for the symmetry of its distribution. The value for a probe was calculated as an average $\mathrm{X}$, taking into account the signals of the neighboring probes (within $1 \mathrm{~kb}$ of the probe). Finally, we defined hypermethylation as a normalized $\log _{2}$ ratio $>0.5$, hypomethylation as a normalized $\log _{2}$ ratio between 0.001 and 0.499 , and unmethylation as a normalized $\log _{2}$ ratio $=0$.

\section{Confirmation of copy number variations}

The accurate quantification of fetal-specific EPs by methylation-based DNA discrimination is of critical importance in the NIPT of fetal T21 using EPs, because such fetal-specific EPs on chromosome 21 are used in the detection of fetal T21 by direct comparison with a placenta-derived DNA methylation marker on a reference chromosome. Therefore, the fetal-specific EPs should be selected from regions without copy number variations $(\mathrm{CNVs})$, which can result in dosage imbalances and interfere with the correct interpretation of results. Therefore, the DNA sequence of the 12 EPs was checked for the presence of CNVs or segmental duplication by searching the Database of Genomic Variants (http://dgv.tcag.ca/dgv/ app/home). The EPs that were free of CNVs and segmental duplications were selected as fetal-specific EPs with potential for the NIPT of fetal T21.

\section{Bisulfite direct sequencing}

We confirmed the MBD array data using bisulfite direct sequencing. DNA samples $(1 \mu \mathrm{g})$ were bisulfite-converted using an EpiTect Bisulfite Kit (Qiagen) according to the manufacturer's instructions. The bisulfite-converted DNA was then amplified by PCR with primers for discrimination of the methylated and unmethylated CpG sites. The sequences of PCR primers are presented in Table 1. PCR reaction solutions contained $10 \mathrm{ng}$ genomic DNA, $10 \mathrm{pM}$ primers, $0.25 \mathrm{mM}$ dNTPs, $1.5 \mathrm{mM} \mathrm{MgCl}_{2}, 1 \mathrm{X}$ buffer, and $0.25 \mathrm{U}$ Taq polymerase per $20 \mu \mathrm{L}$ of total reaction volume. PCR conditions included predenaturation at $95^{\circ} \mathrm{C}$ for $10 \mathrm{~min}, 35 \mathrm{cycles}$ of $95^{\circ} \mathrm{C}$ for $30 \mathrm{sec}, 56^{\circ} \mathrm{C}$ for $30 \mathrm{sec}, 72^{\circ} \mathrm{C}$ for $40 \mathrm{sec}$, and final extension at $72^{\circ} \mathrm{C}$ for $10 \mathrm{~min}$. After PCR amplification, PCR products were purified using a PCR purification kit (Bioneer, Daejeon, Korea) and sequenced using a PRISM BigDye Terminator Cycle Sequencing Kit (Applied Biosystems, Foster city, USA) according to manufacturer's instructions. Sequencing products were analyzed using a PRISM 3100 Genetic Analyzer (Applied Biosystems), and electropherogram traces were interpreted using Genescan software version 3.7 (Applied Biosystems). Corresponding genotypes were assigned using Genotyper software version 3.7 (Applied Biosystems).

\section{Methylation quantification of bisulfite direct sequencing data}

The methylation quantification of bisulfite direct sequencing data was performed as described previously [20]. Briefly, the methylation ratio of each CpG site was calculated as the peak height of $C$ vs. the peak height of $\mathrm{C}$ plus the peak height of $\mathrm{T}$ for each $\mathrm{CpG}$ site, as shown in the computer-generated sequencing chromatogram extracted from the Chromas program (Version 2.32, Technelysium). A single $\mathrm{C}$ at the corresponding $\mathrm{CpG}$ site was considered to be $100 \%$ methylated, a single $\mathrm{T}$ was $100 \%$ unmethylated and overlapping $\mathrm{C}$ and $\mathrm{T}$ was partially methylated (0-100\%).

\section{Noninvasive detection and methylation levels of EPs by qPCR}

We used the PCR conditions described previously [18]. The sequences of PCR primers used are presented in

Table 1 Sequences of primers according to experiment

\begin{tabular}{lll}
\hline Experiment & \multicolumn{2}{l}{ Sequences of primers } \\
\hline $\begin{array}{ll}\text { Bisulfite direct } \\
\text { sequencing }\end{array}$ & EP6 & Forward: 5'-GAT GCG TTA GAT TTA AGG GAG G-3' \\
& Reverse: 5'-CTC ACT CTC ACG AAA CCC CTC-3' \\
& EP7 & Forward: 5'-GAG ATG TाT AGC GTT TGT GGG-3' \\
& Reverse: 5'-AAC TAA TTA CAT AAA ACC CAC CC-3'. \\
qPCR & EP6 & Forward: 5'-TGA ATC AGT TCA CCG ACA GC-3' \\
& Reverse: 5'-GAA ACA ACC TGG CCA TTC TC-3' \\
EP7 & Forward: 5'-CCG TTA TAT GGA TGC CTT GG-3' \\
& Reverse: 5'-AAA CTG TTG GGC TGA ACT GC-3' \\
& EP9 & Forward: 5'-GAC CCA GA CGA TAC CTG GAA-3' \\
& Reverse: 5'- CTG AAC AAA ACT CGG CTT C-3' \\
\hline
\end{tabular}


Table 2 Clinical characteristics of the study population

\begin{tabular}{|c|c|c|c|c|}
\hline Characteristics & $\begin{array}{l}\text { Pregnant women carrying } \\
\text { trisomy } 21 \text { fetuses }(n=12)\end{array}$ & $\begin{array}{l}\text { Pregnant women carrying } \\
\text { normal fetuses }(n=38)\end{array}$ & $\begin{array}{l}\text { Non pregnant women } \\
(n=6)\end{array}$ & $P$ value \\
\hline Age (years) & $32.9 \pm 3.5$ & $35.3 \pm 4.1$ & $29.5 \pm 0.7$ & $0.198^{a}$ \\
\hline Body mass index $\left(\mathrm{kg} / \mathrm{m}^{2}\right)$ & $21.7 \pm 2.5$ & $21.4 \pm 2.6$ & $21.1 \pm 0.3$ & $0.859^{\mathrm{a}}$ \\
\hline Gestational age (weeks) & $11.8 \pm 0.8$ & $12.2 \pm 0.6$ & - & $0.182^{b}$ \\
\hline Gender-ratio of fetus (male: female) & $4: 8$ & $14: 24$ & - & - \\
\hline
\end{tabular}

Data are presented as mean \pm standard deviation.

a one-way analysis of variance test.

$b_{\text {t-test. }}$

Table 1. Each qPCR reaction was performed in a final volume of $20 \mu \mathrm{L}$ with $20 \mathrm{ng}$ of genomic DNA or $5 \mu \mathrm{L}$ of methylated cell free DNA extracted from $1 \mathrm{ml}$ of maternal plasma. We used SYBR Green PCR master mix (Eurogentec, Seraing, Belgium). The amplification program consisted of 3 minutes at $95^{\circ} \mathrm{C}$, followed by 40 cycles of denaturation for 15 seconds at $95^{\circ} \mathrm{C}$ and annealing/extension for 30 seconds at $62^{\circ} \mathrm{C}$. After amplification, melting curve analysis was performed by heating the reaction mixture from 65 to $95^{\circ} \mathrm{C}$ at a rate of $0.5^{\circ} \mathrm{C} /$ second.

For analysis of methylation levels of EPs, the delta $(\Delta)$ threshold cycle $(\mathrm{Ct})$ value was calculated as $\Delta \mathrm{Ct}=\mathrm{Ct}_{\text {input }}$ $\mathrm{Ct}_{\mathrm{MBD}}$. Input denotes the portion without MBD enrichment and MBD denotes the portion after immunoprecipitation. A positive $\Delta \mathrm{Ct}$ value represents a hypermethylated region with enrichment, and a negative $\Delta \mathrm{Ct}$ value represents a hypomethylated region without enrichment.

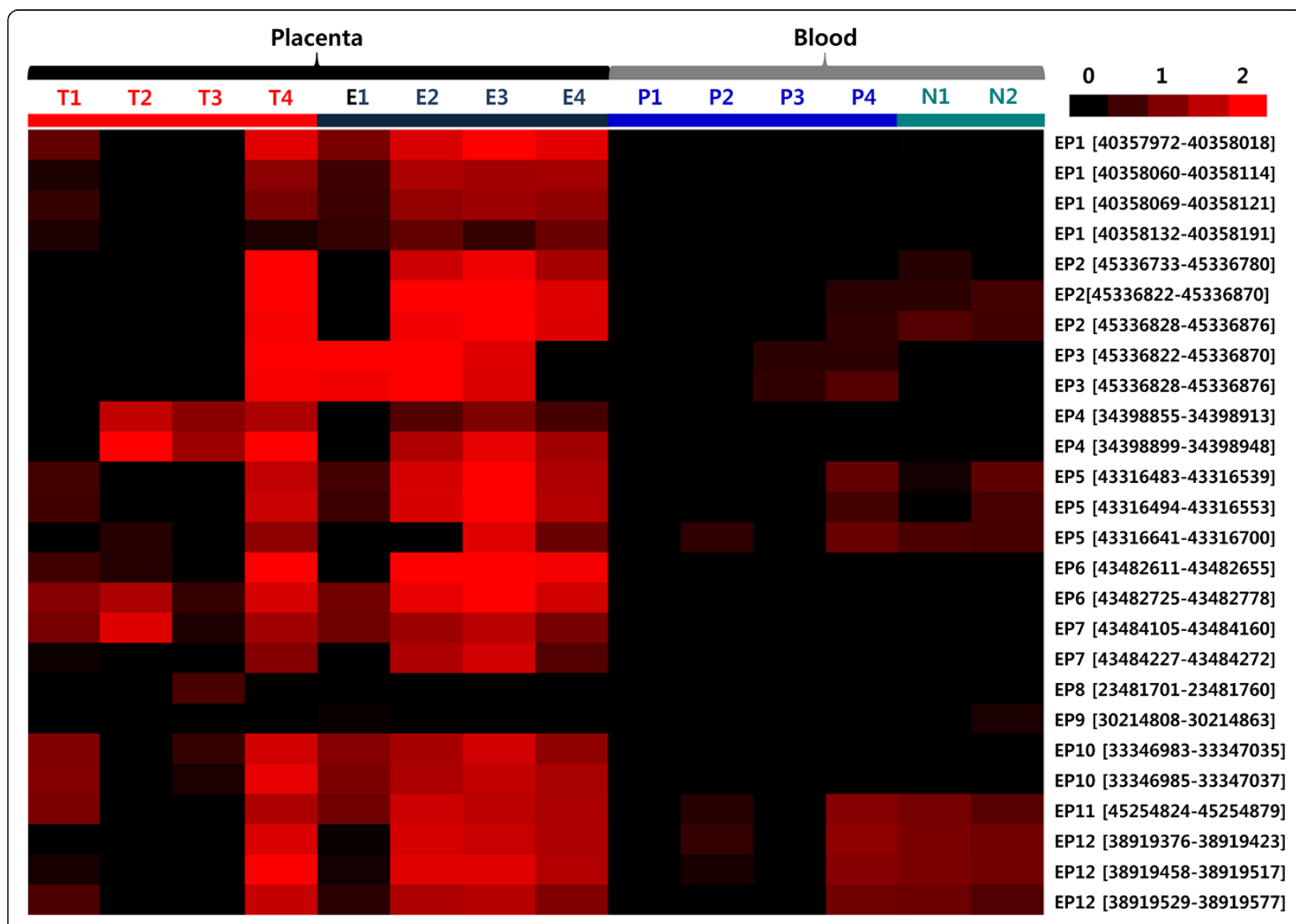

T: Trisomy21 fetus, E: Euploid fetus, P: Pregnant women, N: Non-pregnant women

Figure 1 Methylation levels of EPs in study groups using the tiling oligonucleotide arrays. The red and black colors indicate high expression and non-expression, respectively. 


\section{Statistical analysis}

Data are expressed as a mean \pm standard deviation (S.D.). The clinical data and methylation levels in the study groups were compared using robust one-way analysis of variance (ANOVA), the post hoc Tamhane's T2 test for multiple comparisons, and the t-test for comparisons between the two groups. In all tests, a threshold of $P<0.05$ was set for statistical significance. Statistical analyses were performed using the Statistical Package for Social Sciences 12.0 (SPSS Inc., Chicago, USA).

\section{Results}

\section{Clinical characteristics}

The clinical characteristics of the study groups are shown in Table 2. In total, whole blood samples from non-pregnant euploid women $(n=6)$, whole blood samples and euploid fetal placenta samples from pregnant euploid women $(\mathrm{n}=38)$, and whole blood samples and T21 fetal placenta samples from pregnant euploid women $(n=12)$ were used. At blood sampling, age and body mass index were no differences among all study groups ( $P>0.05$ for both). Maternal age, gestational age, and gender ratio of the fetuses was also not different between pregnant women carrying T21 fetuses and pregnant women carrying normal fetuses $(P>0.05$ for all).

Selection of the fetal-specific EPs for the NIPT of fetal T21 In a high-resolution tiling oligonucleotide array of chromosome 21, whole blood samples from non-pregnant euploid women $(n=2)$, whole blood samples from pregnant euploid women $(\mathrm{n}=4)$, euploid fetal placenta samples $(n=4)$, and T21 fetal placenta samples $(n=4)$ were

Table 3 Methylation levels of EPs according to tissue type

\begin{tabular}{|c|c|c|c|c|c|c|}
\hline \multirow[t]{2}{*}{ EP marker } & \multirow{2}{*}{$\begin{array}{c}\text { Probe position } \\
\text { (Start-Stop) }\end{array}$} & \multicolumn{2}{|c|}{ Placenta $\left(\log _{2}\right)$} & \multicolumn{2}{|c|}{ Normal blood $\left(\log _{2}\right)$} & \multirow[t]{2}{*}{$P$ value } \\
\hline & & Trisomy 21 & Normal & Pregnant & Non-pregnant & \\
\hline \multirow[t]{4}{*}{ EP1 } & $40357972-40358018$ & $0.954 \pm 1.087$ & $2.495 \pm 0.662^{\mathrm{d}, \mathrm{e}}$ & $0.000 \pm 0.000$ & $0.037 \pm 0.037$ & 0.006 \\
\hline & $40358060-40358114$ & $0.501 \pm 0.678$ & $1.663 \pm 0.533^{\mathrm{d}, \mathrm{e}}$ & $0.000 \pm 0.000$ & $0.000 \pm 0.000$ & 0.006 \\
\hline & $40358069-40358121$ & $0.513 \pm 0.581$ & $1.496 \pm 0.468^{\mathrm{d}, \mathrm{e}}$ & $0.000 \pm 0.000$ & $0.000 \pm 0.000$ & 0.005 \\
\hline & $40358132-40358191$ & $0.172 \pm 0.173^{a}$ & $0.932 \pm 0.282^{\mathrm{d}, e}$ & $0.000 \pm 0.000$ & $0.000 \pm 0.000$ & 0.000 \\
\hline \multirow[t]{3}{*}{ EP2 } & $45336733-45336780$ & $0.756 \pm 1.309$ & $1.801 \pm 1.081$ & $0.000 \pm 0.000$ & $0.218 \pm 0.218$ & 0.168 \\
\hline & $45336822-45336870$ & $0.766 \pm 1.327$ & $2.247 \pm 1.320$ & $0.128 \pm 0.221$ & $0.671 \pm 0.131$ & 0.143 \\
\hline & $45336828-45336876$ & $0.727 \pm 1.259$ & $2.151 \pm 1.257$ & $0.145 \pm 0.252$ & $0.892 \pm 0.106$ & 0.151 \\
\hline \multirow[t]{2}{*}{ EP3 } & $45336822-45336870$ & $0.766 \pm 1.327$ & $2.247 \pm 1.320$ & $0.263 \pm 0.263$ & $0.000 \pm 0.000$ & 0.123 \\
\hline & $45336828-45336876$ & $0.727 \pm 1.259$ & $2.151 \pm 1.257$ & $0.395 \pm 0.421$ & $0.000 \pm 0.000$ & 0.145 \\
\hline \multirow[t]{2}{*}{ EP4 } & $34398855-34398913$ & $1.650 \pm 1.060^{b}$ & $0.833 \pm 0.547$ & $0.000 \pm 0.000$ & $0.000 \pm 0.000$ & 0.045 \\
\hline & 34398899-34398948 & $2.423 \pm 1.677^{b}$ & $1.681 \pm 1.018$ & $0.000 \pm 0.000$ & $0.000 \pm 0.000$ & 0.063 \\
\hline \multirow[t]{3}{*}{ EP5 } & $43316483-43316539$ & $0.775 \pm 0.930$ & $2.116 \pm 0.842$ & $0.297 \pm 0.515$ & $0.685 \pm 0.428$ & 0.072 \\
\hline & $43316494-43316553$ & $0.786 \pm 0.972$ & $2.135 \pm 0.914$ & $0.207 \pm 0.358$ & $0.470 \pm 0.392$ & 0.057 \\
\hline & $43316641-43316700$ & $0.538 \pm 0.683$ & $0.979 \pm 1.097$ & $0.446 \pm 0.509$ & $0.871 \pm 0.029$ & 0.814 \\
\hline \multirow[t]{2}{*}{ EP6 } & $43482611-43482655$ & $1.159 \pm 1.335$ & $2.887 \pm 1.844$ & $0.000 \pm 0.000$ & $0.000 \pm 0.000$ & 0.070 \\
\hline & $43482725-43482778$ & $1.624 \pm 0.792^{b, c}$ & $2.405 \pm 0.651^{d, e}$ & $0.000 \pm 0.000$ & $0.000 \pm 0.000$ & 0.001 \\
\hline \multirow[t]{2}{*}{ EP7 } & $43484105-43484160$ & $1.505 \pm 0.922^{b, c}$ & $1.695 \pm 0.366^{d, e}$ & $0.000 \pm 0.000$ & $0.000 \pm 0.000$ & 0.001 \\
\hline & $43484227-43484272$ & $0.430 \pm 0.656$ & $1.384 \pm 0.964^{d}$ & $0.000 \pm 0.000$ & $0.000 \pm 0.000$ & 0.092 \\
\hline EP8 & $23481701-23481760$ & $0.215 \pm 0.372$ & $0.000 \pm 0.000$ & $0.000 \pm 0.000$ & $0.000 \pm 0.000$ & 0.525 \\
\hline EP9 & $30214808-30214863$ & $0.000 \pm 0.000$ & $0.036 \pm 0.062$ & $0.000 \pm 0.000$ & $0.167 \pm 0.167$ & 0.165 \\
\hline \multirow[t]{2}{*}{ EP10 } & $33346983-33347035$ & $1.157 \pm 0.930$ & $1.937 \pm 0.338^{\mathrm{d}, \mathrm{e}}$ & $0.000 \pm 0.000$ & $0.000 \pm 0.000$ & 0.005 \\
\hline & $33346985-33347037$ & $1.170 \pm 1.065$ & $1.951 \pm 0.301^{\mathrm{d}, e}$ & $0.000 \pm 0.000$ & $0.000 \pm 0.000$ & 0.010 \\
\hline EP11 & $45254824-45254879$ & $0.872 \pm 0.896$ & $2.002 \pm 0.406$ & $0.511 \pm 0.642$ & $1.212 \pm 0.173$ & 0.088 \\
\hline \multirow[t]{3}{*}{ EP12 } & $38919376-38919423$ & $0.639 \pm 1.106$ & $1.762 \pm 0.963$ & $0.590 \pm 0.700$ & $1.393 \pm 0.050$ & 0.361 \\
\hline & $38919458-38919517$ & $0.814 \pm 1.249$ & $1.911 \pm 0.983$ & $0.471 \pm 0.651$ & $1.412 \pm 0.073$ & 0.317 \\
\hline & $38919529-38919577$ & $0.812 \pm 0.948$ & $1.556 \pm 0.651$ & $0.329 \pm 0.570$ & $1.122 \pm 0.165$ & 0.256 \\
\hline
\end{tabular}

Data are presented as mean \pm standard deviation. Levels were compared using robust one-way analysis of variance (ANOVA), the post hoc Tamhane's T2 test for multiple comparisons. In post hoc analysis, statistical significance $(P<0.05)$ is presented as follows: ${ }^{\text {a Trisomy }} 21$ placenta versus normal placenta; ${ }^{\mathrm{b}}$ Trisomy 21 placenta versus pregnant women blood; ${ }^{\mathrm{C}}$ Trisomy 21 placenta versus non pregnant women blood; ${ }^{\mathrm{d}}$ Normal placenta versus pregnant women blood; ${ }^{\mathrm{e}}$ Normal placenta versus non pregnant women blood. 
used. Whole blood samples from pregnant euploid women and euploid fetal placental samples were paired. The array data from the 12 EPs in each sample are presented in Figure 1 and their methylation levels are given in Table 3. Among the 12 EPs, ten EPs (EP1-EP7 and EP10-EP12) were identified in probe position of our array and the other two EPs (EP8 and EP9) were located in regions vary near the probe position. Among the confirmed 12 EPs, four EPs (EP2, EP5, EP11, and EP12) were completely methylated in whole blood samples from non-pregnant women, whereas two EPs (EP3 and EP4) were completely unmethylated in one of four euploid placenta samples (Figure 1). Moreover, these EPs showed various methylation discrepancies among the T21 placenta samples that were tested. EP8 and EP9 showed a hypomethylation pattern as a whole, regardless of tissue types and the presence or absence of disease (Figure 1 and Table 3). Thus, only four EPs (EP1, EP6, EP7, and EP10) were confirmed to be hypermethylated in placental samples and hypomethylated in peripheral blood, regardless of pregnancy status (Table 3). However, EP1 was completely unmethylated in two cases among the T21 placenta samples (Figure 1). Moreover, the methylation levels of EP1 in probe regions showed significant differences between T21 placenta samples and normal placenta samples (Table 3).

The CNVs of the 12 EPs is presented in Table 4. Six of 12 EPs (EP2, EP3, EP5, EP8, EP10, and EP11) were located within chromosomal regions with CNVs. Among the four EPs (EP1, EP6, EP7, and EP10) showing tissuespecific epigenetic characteristics between placenta and blood, EP10 was included within chromosomal regions with CNVs which can cause incorrect interpretation of results. Finally, EP6 and EP7 were selected as potentially fetal-specific EPs for the NIPT of fetal T21.

\section{Confirmation of tiling oligonucleotide array data using bisulfite direct sequencing}

We confirmed that the CpG sites of EP6 and EP7 were hypermethylated in placenta compared with blood using bisulfite direct sequencing (Figures 2 and 3). Whole blood samples from non-pregnant euploid women $(n=4)$, whole blood samples from pregnant euploid women $(\mathrm{n}=8)$, euploid fetal placenta samples $(\mathrm{n}=6)$, and T21 fetal placenta samples $(n=5)$ were used. The CpG sites of EP6 and EP7 showed a hypermethylated pattern in placenta compared with blood, regardless of the presence or absence of disease and pregnancy (Figure 4). The methylation ratio in each EP6 and EP7 CpG site was significantly increased in placental samples compared with blood ( $P<0.001$ for all $\mathrm{CpG}$ sites). However, methylation ratio between T21 and normal placental samples was not different $(P>0.05$ for all CpG sites).

\section{Confirmation of tiling oligonucleotide array data using qPCR}

We investigated the tissue-specific methylation levels of EP in whole blood samples from non-pregnant euploid women $(n=6)$, whole blood samples from pregnant euploid women $(n=16)$, euploid fetal placenta samples $(\mathrm{n}=14)$, and T21 fetal placenta samples $(\mathrm{n}=11)$ using qPCR (Table 5). The $\Delta$ Ct of EP6 and EP7 in whole blood samples from non-pregnant women and pregnant women carrying euploid fetuses showed a negative value and presented a hypomethylated pattern of EPs, whereas the $\Delta \mathrm{Ct}$ of the EP6 and EP7 in euploid and T21 placenta samples showed a positive value and presented a hypermethylated pattern of EPs. The $\Delta \mathrm{Ct}$ of the EP6 and EP7 were significantly higher in T21 and euploid placenta samples than in whole blood samples from pregnant women carrying

Table 4 Copy number variation of EPs

\begin{tabular}{|c|c|c|c|}
\hline \multirow[t]{2}{*}{ EP } & \multicolumn{2}{|c|}{ Position of EPs according to assembly } & \multirow{2}{*}{$\begin{array}{l}\text { Copy number variation } \\
\text { (Database of genomic variant) }\end{array}$} \\
\hline & NCBI36/hg18 & GRCh37/hg19 & \\
\hline EP1 & $39279856-39280004$ & $40357986-40358134$ & \\
\hline EP2 & $44161178-44161323$ & $45336750-45336895$ & Variation_7327 (Loss) \\
\hline EP3 & $44161239-44161371$ & $45336811-45336943$ & Variation_7327 (Loss) \\
\hline EP4 & $33320735-33320829$ & $34398865-34398959$ & \\
\hline EP5 & $42189557-42189683$ & $43316488-43316614$ & Variation_10602 (Loss) \\
\hline EP6 & $42355712-42355815$ & $43482643-43482746$ & \\
\hline EP7 & $42357215-42357341$ & $43484146-43484272$ & \\
\hline EP8 & $22403649-22403792$ & $23481778-23481921$ & Variation_30142 (Loss) \\
\hline EP9 & $29136735-29136844$ & $30214864-30214973$ & \\
\hline EP10 & $32268843-32268943$ & $33346972-33347072$ & Variation_4114 (Loss/Gain) \\
\hline EP11 & $44079235-44079322$ & $45254807-45254894$ & $\begin{array}{l}\text { Variation_5162 (Loss),Variation_7327(Loss), } \\
\text { Variation_73647 (Loss), Variation_79379 (Gain) } \\
\text { Variation_90815 (Loss), Variation_90816 (Loss) }\end{array}$ \\
\hline EP12 & $37841284-37841411$ & $38919414-38919541$ & \\
\hline
\end{tabular}




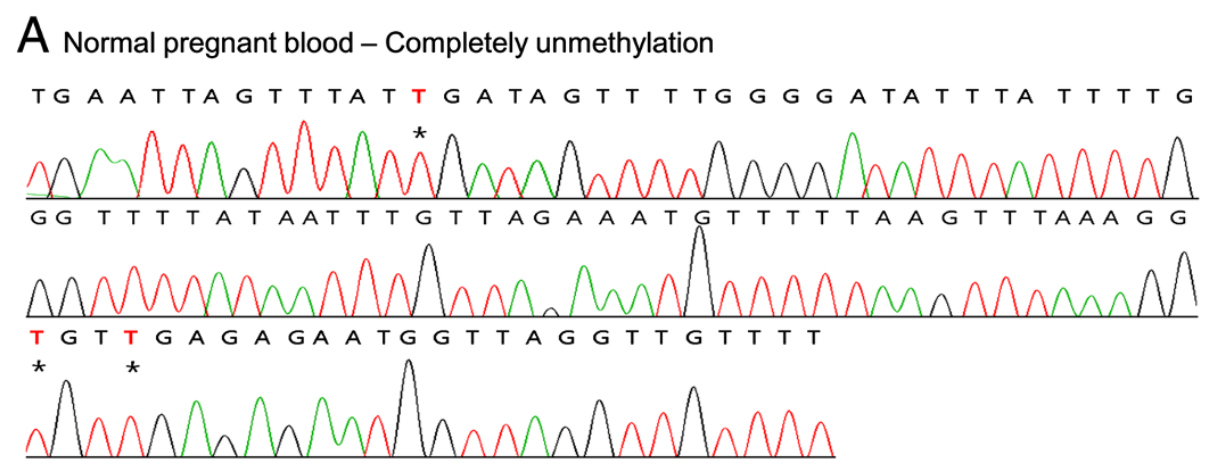

\section{B Normal placenta - Hypermethylation}
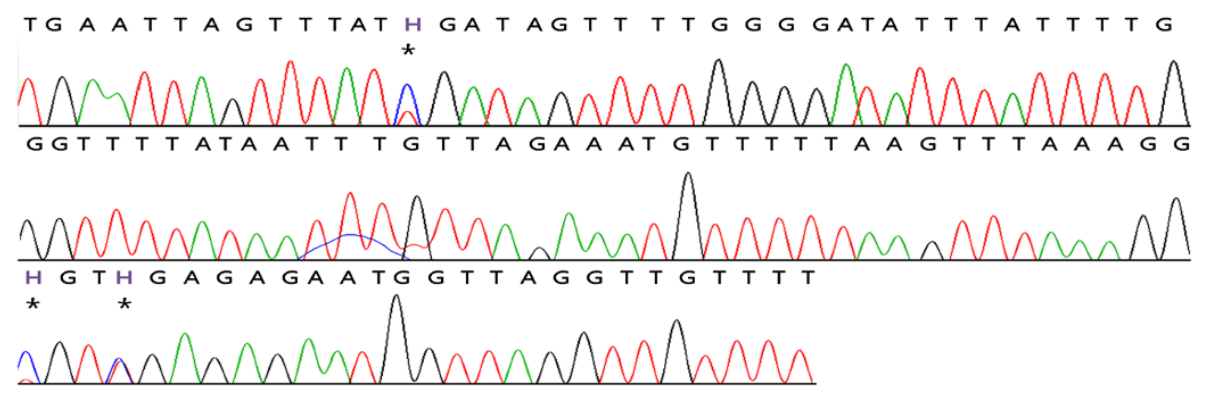

C Trisomy 21 placenta - Hypermethylation

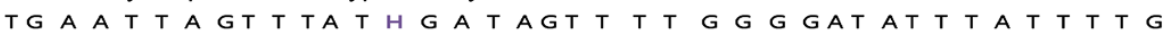

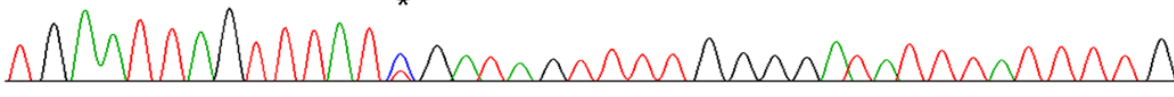

GGT T T T A A A T T T G T A GA A A T G T T T T A A G T T T AAA G G

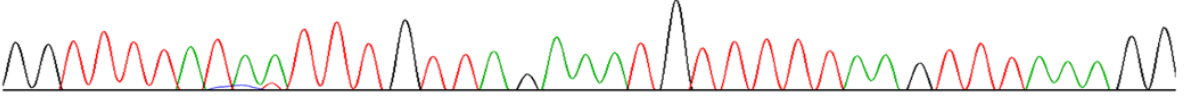

HGTHGAGAGAATGGTTAGGTTGTTTT

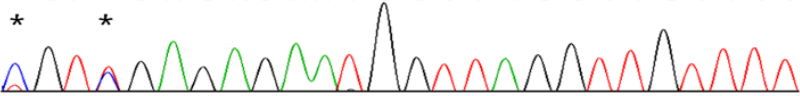

Figure 2 Bisulfite direct sequencing of EP6. The asterisk represents CpG sites in EP6. Red and blue peaks in sequences indicate T and C bases, respectively. Red $(\mathrm{T})$ and violet $(\mathrm{H})$ characters represent unmethylated cytosine and heterozygous cytosine, respectively, in CpG sites. CpG sites of EP6 were completely unmethlylated in normal pregnant blood (A) and were hypermethylated in both normal placenta (B) and trisomy 21 placenta (C).

euploid fetuses and non-pregnant women $(P<0.001$ for all). In addition, we selected EP9 as a negative marker based on array data and investigated its methylation levels (Table 5). The $\triangle \mathrm{Ct}$ of EP9 showed a negative value and presented a hypomethylated pattern in all cases, regardless of the presence or absence of disease and pregnancy. There was no statistically significant difference.

Noninvasive detection of selected EPs in maternal plasma We investigated possibility for noninvasive detection of selected EPs in maternal plasma using qPCR. The maternal plasma samples from 20 pregnant euploid women were used. EP6, EP7, and EP9 levels were measured in all samples without failure of MBD capture. Ct value of EP6, EP7, and EP9 were $29.4 \pm 1.6,29.5 \pm 1.5$, and $34.8 \pm 4.6$, respectively. There was no statistically significant difference between $\mathrm{Ct}$ values of EP6 and EP7 $(P=0.902)$. However, $C t$ value of EP9 was significantly higher than those of EP6 and EP7 $(P<0.001$, Table 5$)$.

\section{Discussion}

In this study, we validated that EP6 and EP7 have a hypermethylated pattern in placenta compared with blood, regardless of the presence or absence of disease and pregnancy and are detectable in maternal plasma. Our results demonstrate that the other ten EPs showed methylation discrepancies that are opposite previous microarray data, which were obtained using whole blood from non-pregnant women and euploid placental samples or were located in CVN regions which can result in 
A Normal pregnant blood - Completely unmethylation

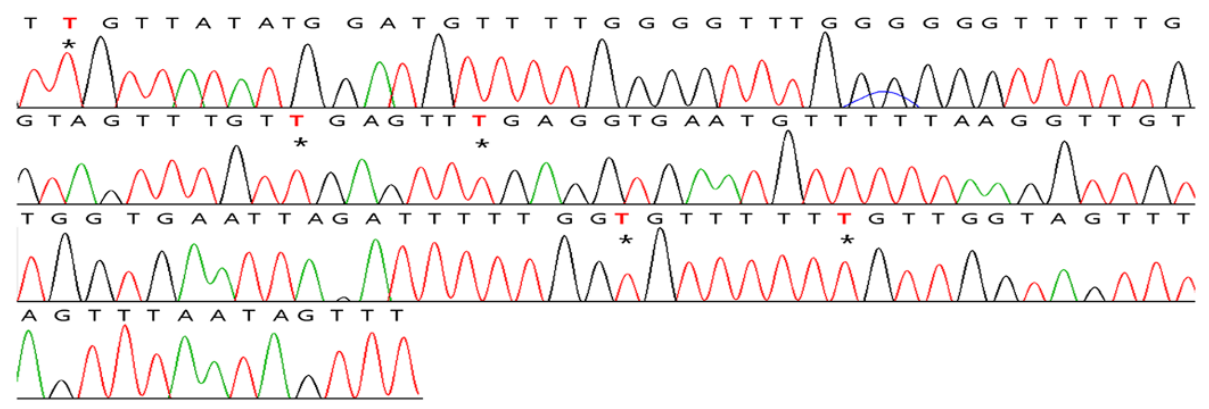

B Normal placenta - Hypermethylation

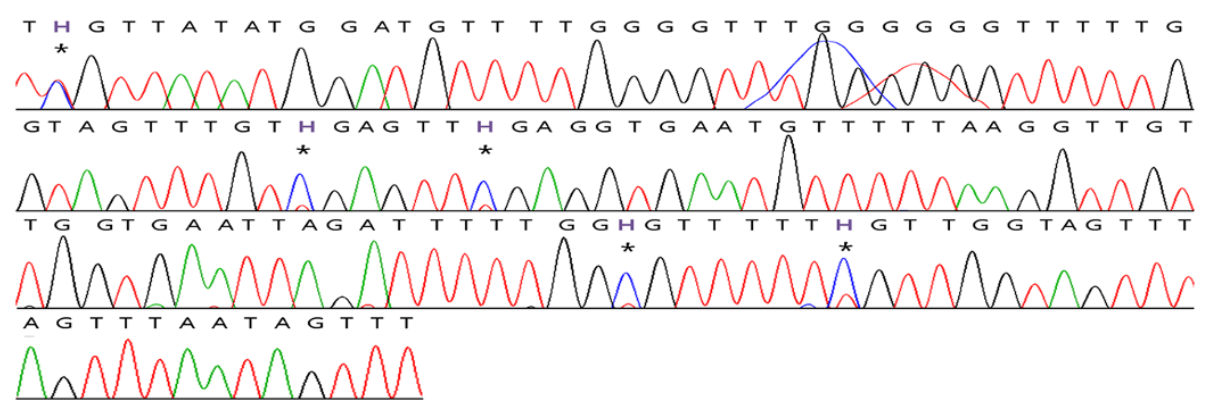

C Trisomy 21 placenta - Hypermethylation

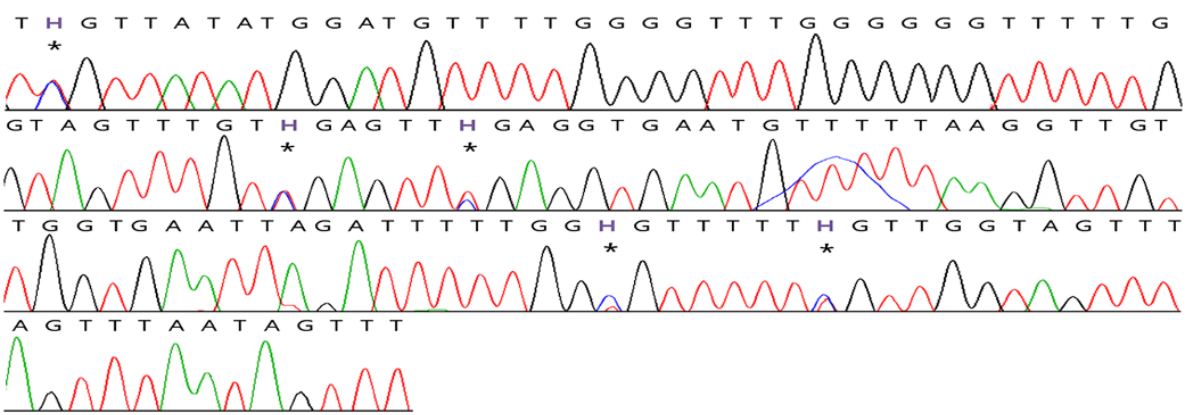

Figure 3 Bisulfite direct sequencing of EP7. The asterisk represents CpG sites in EP7. Red and blue peaks in sequences indicate T and C bases, respectively. Red $(\mathrm{T})$ and violet $(\mathrm{H})$ characters represent unmethylated cytosine and heterozygous cytosine, respectively, in CpG sites. CpG sites of EP7 were completely unmethlylated in normal pregnant blood (A) and were hypermethylated in both normal placenta (B) and trisomy 21 placenta (C).

dosage imbalances. Therefore, we suggest that EP6 and EP7 have potential as effective biomarkers for the noninvasive detection of fetal T21, and that disease-specific epigenetic characteristic should be considered for development of fetal-specific EPs for the NIPT of fetal T21.

In general, $5^{\prime}$-methylcytosine accounts for approximately $1 \%$ of total DNA bases in humans, and therefore potentially affects $70-80 \%$ of all CpG dinucleotides in the genome [21]. Moreover, DNA methylation patterns are dynamic and vary during development and across the genome. DNA methylation has been a topic of considerable interest in development of biomarkers for prognosis and diagnosis of various human diseases including a variety of tumors, Alzheimer's disease, T21, and others [22-25], because DNA methylation has disease-specific epigenetic characteristics that vary in the same tissue according to the presence or absence of disease. In a previous study investigating the alteration of DNA methylation depending on T21 disease status, DNA methylation in total peripheral blood leukocytes and T-lymphocytes from patients with T21 and normal controls has been profiled and found gene-specific abnormalities of CpG methylation in patients with T21 [22]. Therefore, to understand disease-specific epigenetic characteristics it is important to develop effective EPs for prognosis and diagnosis of disease.

Recently, the use of epigenetic differences between maternal whole blood and placental DNA has become 

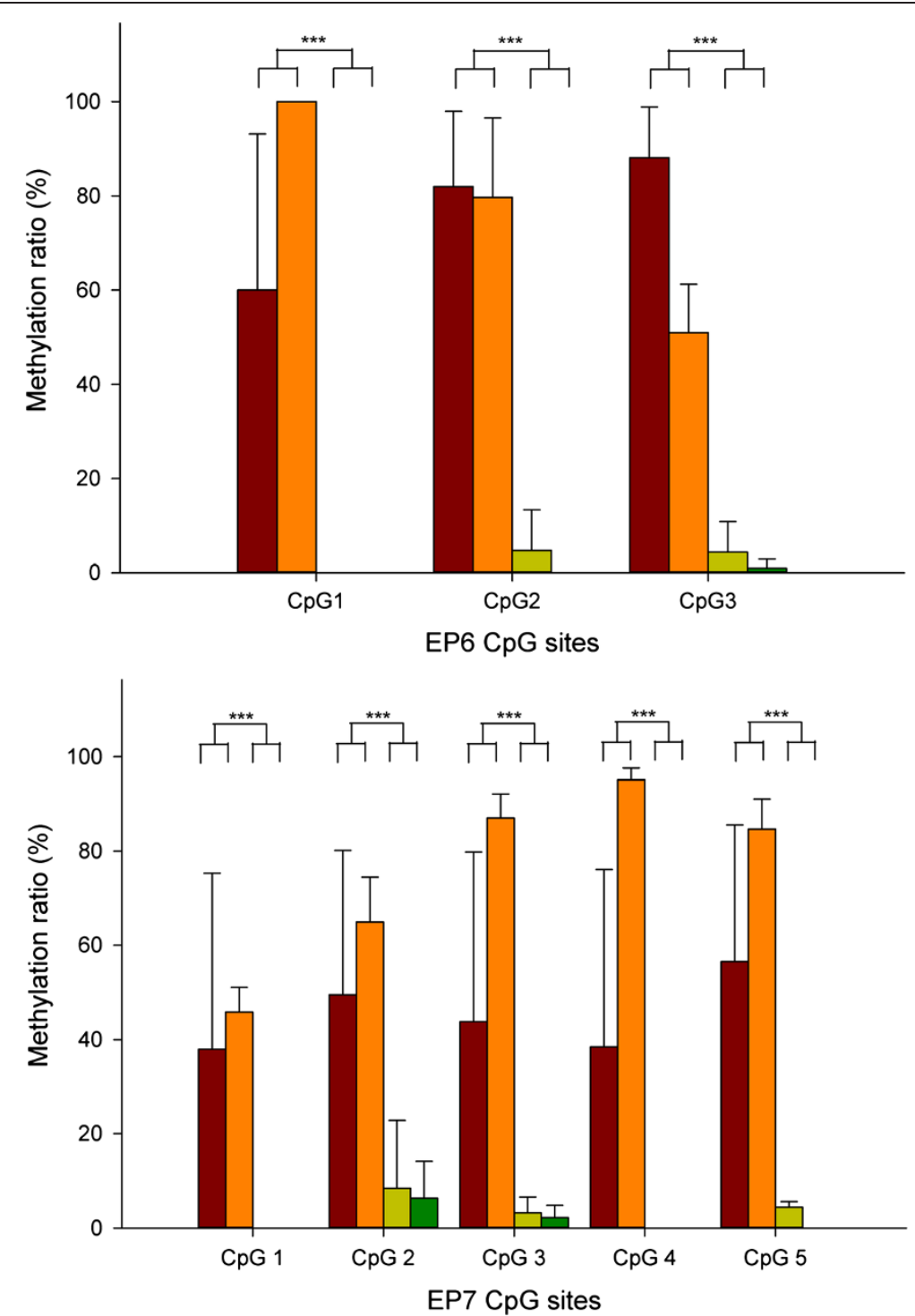

Figure 4 Methylation ratio of each CpG site in EP6 and EP7 by bisulfite direct sequencing. Data are presented as mean \pm standard deviation. Red bars, Trisomy 21 placenta samples $(n=5)$; orange bars, normal placenta samples $(n=6)$; yellowish bars, pregnant normal whole blood $(n=8)$; green bars, non-pregnant normal whole blood $(n=4)$. ${ }^{* *} P<0.001$.

key area of interest for the development of the NIPT of fetal T21. The quantification of fetal DNA by methylation-based DNA discrimination has been used for the NIPT of fetal T21 and promising results have been reported [16-18]. Such fetal-specific EPs on chromosome 21 are applied for the detection of fetal T21 by direct comparison with a placenta-derived DNA methylation marker on a reference chromosome. Theoretically, the methylation ratio of fetal-specific EPs may present equal signal intensity for unaffected fetuses and an increased signal intensity of chromosome 21 for T21 fetuses, because T21 is caused by an extra copy of all or part of chromosome 21. To do that, fetal-specific EPs on chromosome 21 should be hypermethylated in the placenta and unmethylated in the blood, regardless of the presence or absence of pregnancy and T21. However, until now, fetal-specific EPs for the NIPT of fetal T21 have been identified based on the tissue-specific epigenetic characteristics between placenta and maternal blood. The EPs analyzed in this study were also markers identified by the only tissue-specific epigenetic characteristics between whole blood from non-pregnant women $(n=5)$ and euploid placenta samples (first trimester: $\mathrm{n}=3$, third trimester: $\mathrm{n}=2$ ) in a previous study [19]. Epigenetic alteration of these EPs according to the presence or absence of pregnancy and disease has not been validated. Nevertheless, the new platform using the methylation ratio of the EPs has been exhibited excellent clinical 
Table 5 Ct values of EPs by qPCR

\begin{tabular}{|c|c|c|c|c|c|c|c|c|}
\hline \multirow[t]{2}{*}{ EP } & & \multicolumn{2}{|c|}{ Placenta } & \multicolumn{2}{|c|}{ Blood } & \multirow[t]{2}{*}{$P$ value } & \multirow{2}{*}{$\begin{array}{c}\mathrm{Ct}_{\mathrm{MBD}} \text { of cff-DNA } \\
(\mathrm{n}=20)\end{array}$} & \multirow[t]{2}{*}{$P$ value } \\
\hline & & Trisomy $21(n=11)$ & Normal $(n=14)$ & Pregnant $(n=16)$ & Non-pregnant $(n=6)$ & & & \\
\hline \multicolumn{9}{|c|}{ EP6 } \\
\hline & $\mathrm{Ct}_{\text {input }}$ & $22.3 \pm 0.4$ & $23.4 \pm 1.5$ & $23.1 \pm 2.3$ & $22.3 \pm 0.1$ & 0.364 & $29.4 \pm 1.6^{\dagger}$ & 0.021 \\
\hline & $\mathrm{Ct}_{\mathrm{MBD}}$ & $20.9 \pm 0.9^{a, b}$ & $20.9 \pm 1.0^{c, d}$ & $29.3 \pm 1.1$ & $29.1 \pm 2.3$ & $<0.001$ & & \\
\hline & $\Delta \mathrm{Ct}$ & $1.5 \pm 0.9^{a, b}$ & $2.5 \pm 0.9^{c, d}$ & $-6.2 \pm 2.2$ & $-6.7 \pm 2.4$ & $<0.001$ & & \\
\hline \multicolumn{9}{|c|}{ EP7 } \\
\hline & $\mathrm{Ct}_{\text {input }}$ & $22.8 \pm 0.9$ & $24.8 \pm 2.5$ & $24.0 \pm 2.7$ & $21.8 \pm 0.6$ & 0.143 & $29.5 \pm 1.5^{\#}$ & \\
\hline & $\mathrm{Ct}_{\mathrm{MBD}}$ & $20.6 \pm 0.6^{a, b}$ & $21.4 \pm 0.8^{c, d}$ & $29.3 \pm 0.9$ & $27.4 \pm 1.7$ & $<0.001$ & & \\
\hline & $\Delta C t$ & $2.2 \pm 1.1^{a, b}$ & $3.4 \pm 2.2^{c, d}$ & $-5.3 \pm 2.9$ & $-5.6 \pm 1.2$ & $<0.001$ & & \\
\hline \multicolumn{9}{|c|}{ EP9 } \\
\hline & $\mathrm{Ct}_{\text {input }}$ & $20.7 \pm 0.2$ & $21.9 \pm 0.6$ & $21.2 \pm 0.6$ & $22.9 \pm 0.1$ & 0.243 & $34.8 \pm 4.6$ & \\
\hline & $\mathrm{Ct}_{\mathrm{MBD}}$ & $28.8 \pm 0.6$ & $28.9 \pm 1.2$ & $27.0 \pm 1.7$ & $28.6 \pm 2.0$ & 0.469 & & \\
\hline & $\Delta \mathrm{Ct}$ & $-8.1 \pm 0.4$ & $-7.1 \pm 0.8$ & $-5.8 \pm 2.1$ & $-5.6 \pm 2.1$ & 0.421 & & \\
\hline
\end{tabular}

Data are presented as mean \pm standard deviation. $\mathrm{Ct}_{\text {input }}$ denotes the threshold cycle of the input portion without methylated $\mathrm{DNA}$ enrichment. $\mathrm{Ct}_{\mathrm{MBD}}$ denotes the threshold cycle of the methylated DNA enriched portion. The delta $(\Delta)$ threshold cycle $(\mathrm{Ct})$ value was calculated as $\Delta \mathrm{Ct}_{\mathrm{t}}=\mathrm{Ct}_{\mathrm{input}}-\mathrm{Ct}_{\mathrm{MBD}}$. The values were compared using robust one-way analysis of variance (ANOVA), the post hoc Tamhane's T2 test for multiple comparisons. In post hoc analysis, statistical significance $(P<0.05)$ is presented as follows: ${ }^{\mathrm{a}}$ Trisomy 21 placenta versus pregnant women blood; ${ }^{\mathrm{b}}$ Trisomy 21 placenta versus non pregnant women blood; ${ }^{\mathrm{C}}$ Normal placenta versus pregnant women blood; ${ }^{d}$ Normal placenta versus non pregnant women blood; ${ }^{\dagger}$ EP6 versus EP9; ${ }^{\#}$ EP7 versus EP9.

performance (both the sensitivity and specificity were 100\%) $[18,26]$. However, Tong et al. reported a low diagnostic accuracy (a sensitivity of $33 \%$ and a specificity of $73 \%$ ), in contrast to the perfect classification reported previously [27]. In this study, we found that most EPs, except EP6 and EP7, showed discrepant methylation patterns among the tested samples according to the presence or absence of pregnancy and T21. Furthermore, only one T21 case seemed methylation pattern similar to normal cases. This indicates that most EPs identified in a previous study are likely to lead to inaccurate results in non-invasive detection of fetal T21. Therefore, the reproducibility and precision in a realistic clinical setting of using fetal-specific EPs that do not have diseasespecific epigenetic characteristics is controversial. More research is needed to confirm utility of fetal-specific EPs for the NIPT of fetal T21. This study was also limited by its small sample size and the inclusion of only Korean subjects; therefore, a larger-scale study within different ethnic populations is needed. Moreover, EPs based on diseasespecific epigenetic characteristics need to be identified for the NIPT of fetal T21.

\section{Conclusion}

In conclusion, we confirmed that EP6 and EP7 had potential for use as fetal-specific EPs for the NIPT of fetal T21, due to their consistent disease-specific epigenetic characteristics. Therefore, we suggest that consideration of disease-specific epigenetic characteristics of fetal EPs should certainly take precedence in the development of effective and reliable EPs for the NIPT of fetal T21.
Eventually, the identification of effective fetal-specific EPs through the consideration of epigenetic characteristics in disease and tissue may reduce the complexity and cost of these test by excluding the use of inappropriate biomarkers that produce imprecise results in the NIPT of fetal T21.

\section{Abbreviations}

T21: Trisomy 21; NIPT: Non-invasive prenatal testing; cff-DNA: Cell-free fetal DNA; EPs: Epigenetic markers; DMRs: Differentially methylated regions; CVS: Chorionic villus sampling; MBD: Methyl-CpG binding domain-based protein; CNVs: Copy number variations.

\section{Competing interests}

The authors have declared that no competing interests exist.

\section{Authors' contributions}

$J H L$ and DEL participated in study design, execution, analysis, and manuscript drafting. HKA, YJH, and MYK collected samples and participated in interpretation of data and critical discussion. DJK participated in experimental execution and analysis. SYP participated in study design, interpretation of data, and manuscript revision. HMR participated in study design, sample collection, manuscript editing and critical discussion. All authors read and approved the final manuscript.

\section{Acknowledgements}

This study was supported by a grant of the Korean Health Technology R\&D Project, Ministry of Health \& Welfare, Republic of Korea (A111550). The funders had no role in study design, data collection and analysis, decision to publish, or preparation of the manuscript. We thank the following physicians and their associated staff who took the time and effort to participate in this study: Jung Yeol Han, Jin Hoon Chung, Si Won Lee, Jin Woo Kim, Bom Yi Lee, Ju Yeon Park, Eun Young Choi, So Min Seo, Yeon Woo Lee, Hyun Jin Kim, Shin Young Lee, Ah Rum Oh, and Kyoung Soon Kim.

Received: 2 October 2013 Accepted: 5 January 2014

Published: 8 January 2014 


\section{References}

1. Grimshaw GM, Szczepura A, Hultén M, MacDonald F, Nevin NC, Sutton F, Dhanjal S: Evaluation of molecular tests for prenatal diagnosis of chromosome abnormalities. Health Technol Assess 2003, 7:1-146.

2. Mégarbané A, Ravel A, Mircher C, Sturtz F, Grattau Y, Rethoré MO, Delabar $J \mathrm{M}$, Mobley WC: The 50th anniversary of the discovery of trisomy 21: the past, present, and future of research and treatment of Down syndrome. Genet Med 2009, 11:611-616.

3. ACOG Committee on Practice Bulletins: ACOG practice bulletin no. 77: screening for fetal chromosomal abnormalities. Obstet Gynecol 2007, 109:217-227.

4. Malone FD, Canick JA, Ball RH, Nyberg DA, Comstock CH, Bukowski R, Berkowitz RL, Gross SJ, Dugoff L, Craigo SD, Timor-Tritsch IE, Carr SR, Wolfe HM, Dukes K, Bianchi DW, Rudnicka AR, Hackshaw AK, Lambert-Messerlian G, Wald NJ, D'Alton ME, First- and Second-Trimester Evaluation of Risk (FASTER) Research Consortium: First-trimester or second-trimester screening, or both, for Down's syndrome. N Engl J Med 2005, 353:2001-2011.

5. Nicolaides KH: Nuchal translucency and other first-trimester sonographic markers of chromosomal abnormalities. Am J Obstet Gynecol 2004, 191:45-67.

6. Rozenberg P, Bussières L, Chevret S, Bernard JP, Malagrida L, Cuckle H, Chabry C, Durand-Zaleski I, Bidat L, Lacroix I, Moulis M, Roger M, Jacquemot MC, Bault JP, Boukobza P, Boccara P, Vialard F, Giudicelli Y, Ville Y: Screening for Down syndrome using first-trimester combined screening followed by second-trimester ultrasound examination in an unselected population. Am J Obstet Gynecol 2006, 195:1379-1387.

7. American College of Obstetricians and Gynecologists: ACOG practice bulletin No. 88, December 2007. Invasive prenatal testing for aneuploidy. Obstet Gynecol 2007, 110:1459-1467.

8. Alfirevic Z, Sundberg K, Brigham S: Amniocentesis and chorionic villus sampling for prenatal diagnosis. Cochrane Database Syst Rev 2003, 3, CD003252.

9. Lo YM, Corbetta N, Chamberlain PF, Rai V, Sargent IL, Redman CW, Wainscoat JS: Presence of fetal DNA in maternal plasma and serum. Lancet 1997, 350:485-487.

10. Lun FM, Chiu RW, Allen Chan KC, Yeung Leung T, Kin Lau T, Dennis Lo YM: Microfluidics digital PCR reveals a higher than expected fraction of fetal DNA in maternal plasma. Clin Chem 2008, 54:1664-1672.

11. Lo YM, Zhang J, Leung TN, Lau TK, Chang AM, Hjelm NM: Rapid clearance of fetal DNA from maternal plasma. Am J Hum Genet 1999, 64:218-224.

12. Lo YM, Chan KC, Sun H, Chen EZ, Jiang P, Lun FM, Zheng YW, Leung TY, Lau TK, Cantor CR, Chiu RW: Maternal plasma DNA sequencing reveals the genome wide genetic and mutational profile of the fetus. Sci Trans/ Med 2010, 2:61 ra91.

13. Ehrich M, Deciu C, Zwiefelhofer T, Tynan JA, Cagasan L, Tim R, Lu V, McCullough R, McCarthy E, Nygren AO, Dean J, Tang L, Hutchison D, Lu T, Wang H, Angkachatchai V, Oeth P, Cantor CR, Bombard A, Van den Boom $D$ : Noninvasive detection of fetal trisomy 21 by sequencing of DNA in maternal blood: a study in a clinical setting. Am J Obstet Gynecol 2011, 204(205):e1-e11.

14. Bianchi DW, Platt LD, Goldberg JD, Abuhamad AZ, Sehnert AJ, Rava RP: MatErnal BLood IS source to accurately diagnose fetal aneuploidy (MELISSA) study group: genome-wide fetal aneuploidy detection by maternal plasma DNA sequencing. Obstet Gynecol 2012, 119:890-901.

15. Lim JH, Park SY, Ryu HM: Non-invasive prenatal diagnosis of fetal trisomy 21 using cell-free fetal DNA in maternal blood. Obstet Gynecol Sci 2013, 56:58-66.

16. Mersy E, Smits $L$, van Winden $L A$, de Die-Smulders $C E$, South-East Netherlands NIPT C, Paulussen AD, Macville MV, Coumans AB, Frints SG: Noninvasive detection of fetal trisomy 21: systematic review and report of quality and outcomes of diagnostic accuracy studies performed between 1997 and 2012. Hum Reprod Update 2013, 19:318-329.

17. Lim JH, Kim SY, Park SY, Lee SY, Kim MJ, Han YJ, Lee SW, Chung JH, Kim MY, Yang JH, Ryu HM: Non-invasive epigenetic detection of fetal trisomy 21 in first trimester maternal plasma. PLoS One 2011, 6:e27709.

18. Papageorgiou EA, Karagrigoriou A, Tsaliki E, Velissariou V, Carter NP, Patsalis PC: Fetal-specific DNA methylation ratio permits noninvasive prenatal diagnosis of trisomy 21. Nat Med 2011, 17:510-513.

19. Papageorgiou EA, Fiegler H, Rakyan V, Beck S, Hulten M, Lamnissou K, Carter NP, Patsalis PC: Sites of differential DNA methylation between placenta and peripheral blood: molecular markers for noninvasive prenatal diagnosis of aneuploidies. Am J Pathol 2009, 174:1609-1618.

20. Jiang M, Zhang Y, Fei J, Chang X, Fan W, Qian X, Zhang T, Lu D: Rapid quantification of DNA methylation by measuring relative peak heights in direct bisulfite-PCR sequencing traces. Lab Invest 2010, 90:282-290.

21. Ehrlich M, Gama-Sosa MA, Huang LH, Midgett RM, Kuo KC, McCune RA, Gehrke C: Amount and distribution of 5-methylcytosine in human DNA from different types of tissues of cells. Nucleic Acids Res 1982, 10:2709-2721.

22. Kerkel K, Schupf N, Hatta K, Pang D, Salas M, Kratz A, Minden M, Murty V, Zigman WB, Mayeux RP, Jenkins EC, Torkamani A, Schork NJ, Silverman W, Croy BA, Tycko B: Altered DNA methylation in leukocytes with trisomy 21. PLoS Genet 2010, 6:e1001212.

23. Wang SC, Oelze B, Schumacher A: Age-specific epigenetic drift in late-onset Alzheimer's disease. PLoS One 2008, 3:e2698.

24. Kanwal R, Gupta S: Epigenetic modifications in cancer. Clin Genet 2012, 81:303-311.

25. Feinberg AP: Genome-scale approaches to the epigenetics of common human disease. Virchows Arch 2010, 456:13-21.

26. Tsaliki E, Papageorgiou EA, Spyrou C, Koumbaris G, Kypri E, Kyriakou S, Sotiriou C, Touvana E, Keravnou A, Karagrigoriou A, Lamnissou K, Velissariou $\checkmark$, Patsalis PC: MeDIP real-time $\mathrm{qPCR}$ of maternal peripheral blood reliably identifies trisomy 21. Prenat Diagn 2012, 32:996-1001.

27. Tong YK, Chiu RW, Chan KC, Leung TY, Lo YM: Technical concerns about immunoprecipitation of methylated fetal DNA for noninvasive trisomy 21 diagnosis. Nat Med 2012, 18:1327-1328.

doi:10.1186/1755-8794-7-1

Cite this article as: Lim et al:: Disease specific characteristics of fetal epigenetic markers for non-invasive prenatal testing of trisomy 21. BMC Medical Genomics 2014 7:1.

\section{Submit your next manuscript to BioMed Central and take full advantage of:}

- Convenient online submission

- Thorough peer review

- No space constraints or color figure charges

- Immediate publication on acceptance

- Inclusion in PubMed, CAS, Scopus and Google Scholar

- Research which is freely available for redistribution

Submit your manuscript at www.biomedcentral.com/submit
C Biomed Central 